\title{
Medical and Surgical Management of Fecal Incontinence after Repair of High Imperforate Anus Anomalies
}

Talal Abdallah Al-Malki

Additional information is available at the end of the chapter

\section{Introduction}

Fecal incontinence is a socially distressing misfortunate condition, which can be very embarrassing. It affects children, with dramatic behavioral and personality changes, with patients becoming socially withdrawn and reluctant to leave their homes. Victims are housebound and become socially disruptive. This condition may be due to (1) congenital anorectal malformations (2) trauma to anal sphincter (3) injuries to the sphincter following operations on the anal canal and (4) central nervous disease or nerve injury.

It can occur from a variety of conditions. The most common is overflow incontinence from functional fecal retention, but it can also occur in otherwise healthy children with functional nonretentive fecal soiling or in children with organic causes of fecal incontinence, such as congenital malformations, or any other condition affecting the anorectum, anal sphincters, or the spinal cord.

Mild to moderate incontinence, when there is no injury to the sphincters, can be treated by dietary modification, pharmacological treatment and behavioral therapy. Dietary modification entails having foods with high fiber content such as fruits, vegetables, beans and whole wheat bread. Pharmacological treatment includes prescription of synthetic cellulose and psyllium husk derived from the plantago tree, and antidiarrhea drugs such as Loperamide, diphenoxylate hydrochloride and codeine phosphate. Behavioral therapy techniques involve toilet training; urge control strategies, as well as pelvic floor physical therapy and biofeedback.

Surgical management of fecal incontinence should be reserved for patients with identifiable anal sphincter defects. It includes sphincteroplasty, which is indicated for sphincter disruption after surgical procedures, and muscle transpositions procedures, that are recommended when anal incontinence is secondary to anal sphincter disruption unresponsive to repair, neurogenic 
sphincter compromise, or congenital sphincter disorders. Muscle transpositions procedures include graciloplasty and gluteoplasty.

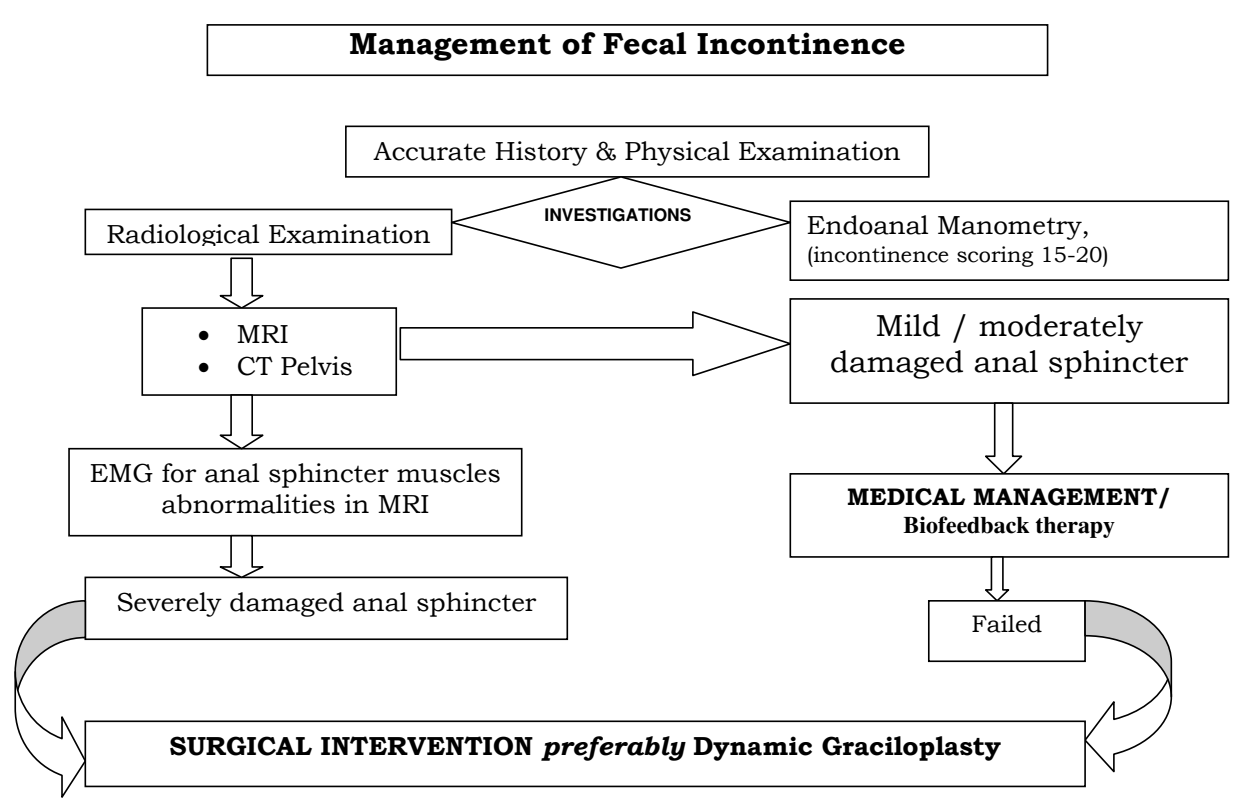

\section{Medical and surgical managementof fecal incontinence}

Graciloplasty was first described in 1952 by Pickrell who carried out gracilis muscle transposition for management in the pediatric population. The majority of muscle fibers in the gracilis are type II fast twitch, easily fatigable as opposed to approximately $80 \%$ of type I slow twitch, fatigue resistant muscle fibers in the external anal sphincter. Beaten and his coworkers introduced neurostimulation of the gracilis with the idea of converting type II muscle fibers to type I fatigue-resistant fibers (dynamic graciloplasty).

Other modalities for managing incontinence include artificial anal sphincter (Acticon Neosphincter $^{\mathrm{TM}}$, American Medical Systems, Minnetonka, MN), which is indicated for patients with anal incontinence secondary to neurologic disorders that affect the sphincter muscles or trauma. This procedure is generally contraindicated in patients with severe diarrhea disorders, poor functional status, or physical and/or mental disease that would limit their ability to adequately use the artificial sphincter. The Secca procedure is another alternative for such patients, where temperature-controlled radio-frequency energy is delivered to the anal musculature through a specially designed anoscopic instrument. It offers a less-invasive option for treatment of fecal incontinence, as compared to surgery, and is performed on an outpatient 


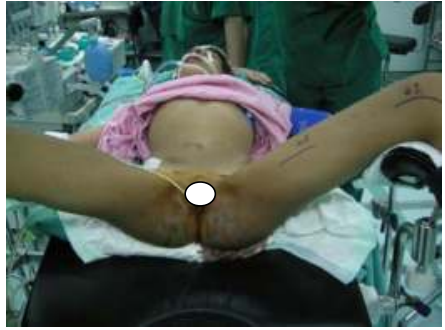

A

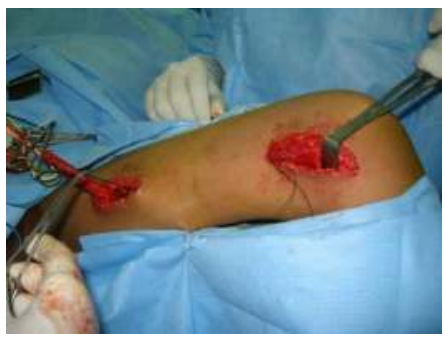

C

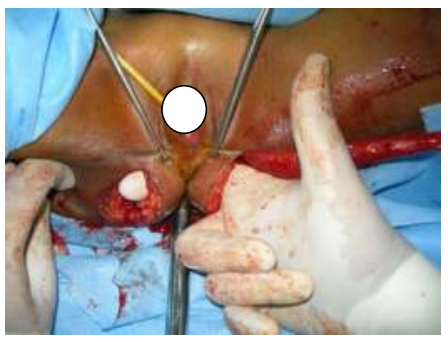

E

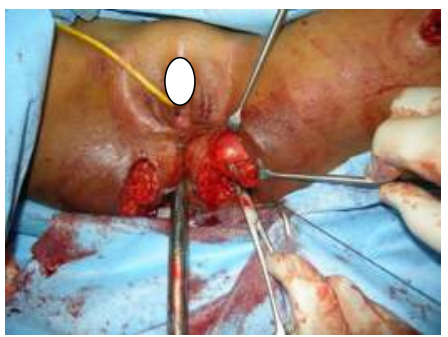

G

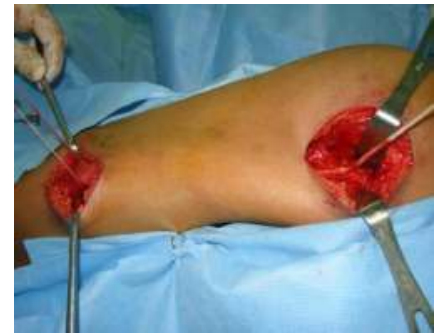

B

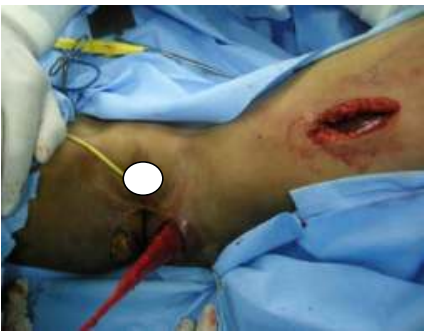

$\mathrm{D}$

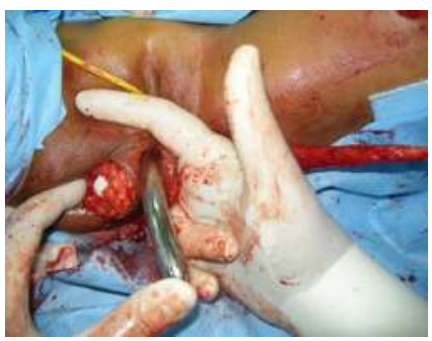

$\mathrm{F}$

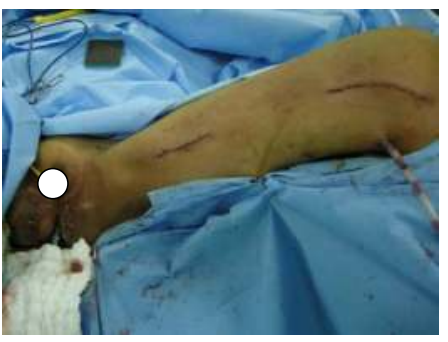

$\mathrm{H}$

Figure 1. Dynamic graciloplasty in a fecal incontinence patient. The surgery was performed in all patients who failed to respond to all non surgical management, in addition to the cases that demonstrated bad condition of the external anal sphincter by MRI, EMG and Manometry. A: Incisions marks before Gracilis muscle dissection; B: Gracilis muscle completely dissected; C: Gracilis muscle pulled out of its bed; D: Gracilis muscle pulled out of its bed; E: tunneling above the anus; F: Gracilis muscle tunneling below the anus; G: Gracilis muscle wrapped around anal canal; $\mathrm{H}$ : Wound closed. 
basis using conscious sedation. Sacral neuro-modulation is another modality for management; the procedure involves the placement of a quadrupolar electrode through the sacral foramina of S3 or S4 to stimulate the pelvic floor musculature. Injectable bulking agents have been used for anal incontinence that results secondary to internal anal sphincter incompetence or disruption. Autologous fat, collagen, Teflon paste and, most recently, injectable silicone particles and PTP (Bioplastique) have been used as bulking agents.

For all patients who undergo the procedure, thorough history taking including the nature of the congenital lesion and the type of surgical intervention should be preformed. The degree of incontinence is usually determined. Waxner and his co-workers graded fecal incontinence in children based on the type of fecal contents, need to wear pad and whether there is alteration in life style and the frequency of occurrence of the afore mentioned factors using a score with a range between 0 (never) and 20 (complete incontinence). Then a meticulous physical examination should be done, with special emphasis on the anal sphincter. Pelvic CT scanning and MRI should be executed to define the status of the sacrum and pelvis, and that of the sphincter complex. EMG for anal sphincter muscles should be performed in the cases that show abnormalities in MRI. Furthermore, anorectal manometry, is to be carried out to quantify the impact of sphincter injury on sphincter function by measures both resting anal sphincter canal tone (internal anal sphincter activity) and squeeze pressure (external sphincter activity) in four quadrants (anterior, right, left, and posterior).

For patients who exhibit severely damaged anal sphincter by MRI, EMG, and endoanal manometry, (incontinence scoring 15-20). These patients should be scheduled to surgery without giving trial to conservative management. The management approach, for other cases, should start with medical treatment and behavioral therapy. Patients who fail to respond properly should be subjected to surgery.

Patients should be instructed to increase dietary fiber contents. They should be told to collect 25-30 gms of dietary fiber per day.

Patients who fail to abide by the regimen should be given the fiber supplements methylcellulose and psyllium husk derived from the plantago tree. In cases with loose stool the antidiarrhea drug Loperamide hydrochloride should be prescribed.

In addition to the above dietary and medical management, behavioral techniques i.e. Toileting techniques and Urge control should be applied in all patients.

Patients who fail to respond to the above management protocol have to be sent to a biofeedback therapist. There they should be subjected to two types of training:

A motor part: This comprised insertion of an anal manometric probe which transform the contractions of the anal sphincter to a visual display, allowing the child to learn how to contract his sphincter properly in an increasing force to overcome fecal soiling.

A sensory part: A manometric balloon probe is to be inserted into the rectum; the balloon is filled with air to train the child to respond to the sensation of rectal filling. 
Patients who are scheduled for surgical intervention are those who failed to respond to all non surgical management, in addition to the cases that demonstrated bad condition of the external anal sphincter by MRI, EMG and manometry.

The technique of graciloplasty is performed by using the left gracilis muscle. The muscle should be dissected till the neurovascular bundle entrance, tunneled and wrapped around the anal canal and sutured to itself using proline stitches. All wounds should be irrigated and closed; a suction drain is placed along the gracilis bed. Postoperatively, both lower limbs should be kept in adduction for three days.

Dynamic graciloplasty entails electric stimulation of the muscle after surgery to transform its fibers to type I slow twitch, fatigue resistant muscle fibers. Chronic low-frequency stimulation should be undertaken in six sessions at 2-week interval over the subsequent 12 weeks. During these sessions, the amount of time during which the muscle been stimulated is to be gradually increased until full-time stimulation is achieved. The conversion of the muscle could be seen in the decreased fusion frequency and the capacity of the muscle to sustain a prolonged contraction. When this status has been achieved, the muscle will show continuous contraction and the anal canal is expected to respond by continuous closure.

The primary step is to assess the degree of incontinence and degree of anal sphincter damage. This is done using a scoring system for incontinence, pelvic MRI, endoanal manometry and EMG. Although endoanal ultra-sonography is very effective to demonstrate the degree of anal sphincter injury, it is sometimes not performed due to the unavailability of an expert sonographer for interpretation of results. The decision taken, for children to be managed surgically from the start, is usually made based on the input from all investigations (continence score 15-20, damaged anal sphincter as illustrated by MRI, Manometry and EMG). Those cases that are treated medically with dietary management and medical treatment. Antidiarrheal drugs are to be used in cases with loose stool.

Patients not responding to medication should be sent to biofeedback therapy. Most cases are expected to show low compliance to the therapy and may refuse to complete the sessions. In general, it is expected that biofeedback in children is discouraging. However, it may show some improvement at early follow up, but not after long term follow up. It has been reported that even demonstrated higher rather than lower rates of persisting symptoms of fecal incontinence up to 12 months when biofeedback is added to conventional treatment. Biofeedback needs not only a skilled therapist, but in addition the will and determination of the patient; which is difficult to achieve in such young ages of patients.

Usually dynamic graciloplasty, is performed following the the conventional operative steps as described in literature. However, the gracilis tendon may be wrapped around the anus and been sutured to itself, instead of suturing it to the opposite ischial tuberosity. Furthermore, one gracilis muscle may be used for repair, while in some other reports both muscles were employed to form a sling around the anal canal simulating the action of the puborectalis. Nonetheless, no superior outcomes were demonstrated to have been achieved as compared to the technique adopted using the gracilis tendon wrapped around the anus. In the later technique short-and medium-term morbidity rates, are lower compared to the conventional 
techniques. Wound seroma that may take place in some cases in the bed of gracilis muscle, may be drained with complete cure outcomes. Wound infections can be managed and cured with drainage and antibiotics according to culture and sensitivity. Medium-term follow up is necessary for patients who may suffer anal stenosis, which is to be treated with a regular schedule of dilatation, and for cases that experience fecal impaction, disimpaction may be performed under anesthesia.

\section{Conclusion}

Children with low incontinence score (5-10) after repair of high imperforate anus anomalies, which show no evidence of anatomical damage of the sphincter, can be treated successfully with medical treatment, while Biofeedback therapy has discouraging results, because it is refused by children. Therefore, cases who fail medical treatment or have high incontinence score (16-20) are recommended to undergo surgical intervention preferably dynamic graciloplasty which proved $100 \%$ success rate and demonstrated excellent results in the operated children.

\section{Author details}

Talal Abdallah Al-Malki

College of Medicine, Taif University, Pediatric Surgery Department, Alhada Armed Forces Hospital, Taif, Kingdom of Saudi Arabia

\section{References}

[1] Pensabene L, Nurko S. Management of fecal incontinence in children without functional fecal retention. Currt Treat Options Gastroenterol 2004; 7:.381-390.

[2] Parker SC, Thorsen A. Fecal incontinence. Surg Clin North Am 2002; 82: 1273-1290.

[3] Scarlett Y. Medical management of fecal incontinence. Gastroenterology 2004; 126: S55-63.

[4] Rudolph W, Galandiuk S. A practical guide to the diagnosis and management of fecal incontinence. Mayo Clin Proc 2002; 77: 271-275.

[5] Norton C. Behavioral management of fecal incontinence in adults. Gastroenterology 2004; 126: S64-S70. 
[6] Corman ML. Colon and Rectal Surgery.5 $5^{\text {th }}$ ed. Philadelphia: Lippincott Williams and Wilkins; 2004.

[7] Sielezneff I, Malouf AJ, Bartolo DC, Pryde A, Douglas S. Dynamic graciloplasty in the treatment of patients with fecal incontinence. Br J Surg 1999; 86: 61-65.

[8] Christiansen J, Hansen CR, Rasmussen O. Bilateral gluteus maximus transposition for anal incontinence. Br J Surg 1995; 82:903-905.

[9] Pickrell KL, Broadbent TR, Masters FW, Metzger JT. Construction of a rectal sphincter and restoration of anal continence by transplanting the gracilis muscle; a report of four cases in children. Ann Surg 1952; 135: 853-862.

[10] Williams NS, Hallan RI, Koeze TH, Pilot MA, Watlens ES. Construction of a neoanal sphincter by transposition of the gracilis and prolonged neuromuscular stimulation for the treatment of fecal incontinence. Ann R Coll Surg Engl 1990; 72: 108-113.

[11] Baeten CG, Bailey HR, Bakka A, Belliveau P, Berg E, Buie WD et al. Safety and efficacy of dynamic graciloplasty for fecal incontinence: report of a prospective, multicenter trial. Dynamic Graciloplasty Therapy Study Group. Dis Colon Rectum 2000; 43: 743-751

[12] Madoff RD, Baeten CG, Christiansen J, Rosen HR, Williams NS, Heine JA. Standards for anal sphincter replacement. Dis Colon Rectum 2000; 43: 135-141.

[13] Takahashi T, Garcia-Osogobio S, Valdovinos MA, Belmonte C, Barreto C, Velasco L. Extended two-year results of radio-frequency energy delivery for the treatment of fecal incontinence (the Secca procedure). Dis Colon Rectum 2003; 46: 711-715.

[14] Matzel KE, Stadelmaier U, Hohenfellner M, Gall FP. Electrical stimulation of sacral spinal nerves for treatment of fecal incontinence. Lancet 1995; 346:1124-1127.

[15] Tjandra JJ, Lim JF, Hiscock R, Rajendra P. Injectable silicone biomaterial for fecal incontinence caused by internal anal sphincter dysfunction is effective. Dis Colon Rectum. 2004; 47: 2138-2146.

[16] Blaisdell PC. Repair of the incontinent sphincter ani. Am J Surg 1957; 94: 573-576.

[17] Rudolph W, Galandiuk S. A practical Guide to the Diagnosis and Management of Fecal Incontinence. Mayo Clin Proc 2002; 77: 271-275.

[18] Loening-Baucke V. Modulation of abnormal defecation dynamics by biofeedback treatment in chronically constipated children with encopresis. J Pediatr 1990; 116: 214-222.

[19] Loening-Baucke V. Biofeedback treatment for chronic constipation and encopresis in childhood: long-term outcome. Pediatrics 1995; 96: 105-110. 
[20] Brazelli M, Griffiths P. Behavioural and cognitive interventions with or without other treatments for the management of fecal incontinence in children. Cochrane Database of Systematic Reviews 2006; 19: CD002240.

[21] Nixon HH. Operation for incontinence following anorectal anomalies: Levatorplasty and gracilis transplantation. Rob and Smith's Operative Surgery colon, rectum and anus. 5th ed. Chapman and Hill; 1993: 460-461.

[22] Madoff RD, Rosen HR, Baeten CG, LaFontaine LJ, Cavina E, Devesa M. Safety and efficacy of dynamic muscle plasty for anal incontinence: lessons from a prospective, multicenter trial. Gastroenterology 1999;116: 549-556.

[23] Thornton MJ, Kennedy ML, Lubowski DZ, King DW. Long-term follow-up of dynamic graciloplasty for fecal incontinence. Colorectal Dis 2004; 6: 470-476.

[24] Wexner SD, Gonzalez-Padron A, Teoh TA, Moon HK. The stimulated gracilis neosphincter for fecal incontinence: a new use for an old concept. Plast Reconsr Surg 98; 1996: 693-699

[25] Wright JJr, Gehrich AP, Albright TS. Mangement of Anal Incontinence. J Pelvic Med Surg 12; 2006: 125-140 